\title{
A Real-time Rheological Measurement for Biopolymer 3D Printing Process
}

\author{
Anchyza Yokpradit, ${ }^{1}$ Teerawat Tongloy, ${ }^{2}$ \\ Supranee Kaewpirom, ${ }^{3}$ and Siridech Boonsang ${ }^{1 *}$ \\ ${ }^{1}$ Department of Electrical Engineering, Faculty of Engineering, \\ King Mongkut's Institute of Technology Ladkrabang, Bangkok 10520, Thailand \\ ${ }^{2}$ Department of Manufacturing System Engineering, College of Advanced Manufacturing Innovation (AMI), \\ King Mongkut's Institute of Technology Ladkrabang, Bangkok 10520, Thailand \\ ${ }^{3}$ Department of Chemistry, Faculty of Science, Burapha University, Chonburi 20131, Thailand
}

(Received December 18, 2017; accepted July 27, 2018)

Keywords: 3D bioprinting, biopolymer fluid dispensing, volume flow rate, tissue engineering, biopolymer, user interface

Biopolymers combined with 3D printing technology for tissue engineering have been extensively used in many biomedical applications such as organ and tissue regeneration. The 3D printing technology generally relies on a continuous dispensing process, which is usually known as complicated dynamic processes. This is primarily caused by the rheological properties of materials. In addition, the primary concern for biopolymer printing is the timedependent variation of material properties such as viscosity during the printing process. The variation of rheological properties significantly affects the printing process especially the volume flow rate control. In general, the measurement of rheological properties is carried out before the dispensing process. The setting up of fluid flow control is usually performed according to parameters derived from the premeasured rheological values. However, such a method may not provide optimized parameters for printing control with some biopolymers. An innovative method of measuring rheological properties is proposed in this paper. An image processing procedure is employed to determine the fluid flow at a certain time in the fluid dispensing process. Experiments were carried out in which the rheological properties of poly(vinyl alcohol) (PVA) and poly-(vinyl alcohol)/chitosan (PVA/CS) aqueous solutions used for dispensing the polymers were evaluated at different time stamps and applied pressures. The measured flow rates were used to construct a pressure-dependent fluid flow rate curve as a function of time to identify the rheological properties of fluid materials under specific times and conditions.

\section{Introduction}

The requirements of organ donors are countless and that reality motivates many tissue engineering researchers to focus on the possibilities of tissue scaffolding. Biopolymers for

\footnotetext{
*Corresponding author: e-mail: siridech.bo@kmitl.ac.th
} https://doi.org/10.18494/SAM.2018.1851 
tissue engineering have been applied in organ and tissue regeneration in combination with 3D printing technology. ${ }^{(1)}$ However, the deviation of material properties during the printing process is the most challenging issue. The conventional method of measuring rheological properties is carried out using a rheometer before the dispensing process. However, the rheological properties of biopolymers are time-dependent. Therefore, inaccurate parameters may be used under certain processing conditions. It also affects the precise control of flow rate, especially in the case of biopolymers with rapid gelation.

The modeling of a fluid dispensing process has been studied to precisely control the droplet volume in some demanding applications such as the soldering of integrated circuits and surface mount technology. Researchers have proposed mathematical models to understand and use for controlling dynamic dispensing processes. ${ }^{(2-11)}$ Process conditions and material properties may significantly affect the fluid dispensing process including the temperature of fluids, the pressure of a compressed air supply, the flow behavior of dispensed fluids, and the structural parameters of the dispensing system. The complexity of material parameters and the variation of syringe pressure in the dispensing process are both time-dependent and may considerably affect the volume of fluid being dispensed. Therefore, the measurement of rheological properties at specific times and under certain conditions is proposed.

In this research, we present a system for the real-time measurement of rheological properties during the dispensing process. The system provides the measurements of accurate rheological parameters of materials at specific times and under certain conditions. This innovative system for the measurement of rheological properties is a combination of a well-organized user interface for setting up measurement and a vision measurement system. To measure the volume of dispensed fluid, an image processing method is applied. The combination of pressure data in a syringe acquired from a pressure sensor and measured volume flow rates is used to construct a pressure-dependent fluid flow rate curve as a function of time. The rheological properties of fluid materials are then determined by a numerical analysis procedure. The results from the numerical analysis provide a time-dependent power law index $(N)$ of fluids. This index can be used as the flow control parameters of dispensing processes.

\section{Materials and Methods}

Both the polymer and biopolymer used in this study are summarized in Table 1. A poly-(vinyl alcohol) (PVA) gel is created by adding $5 \%$ of a crosslinking solution $(2.23 \%$ glutaraldehyde solution in water) to a $10 \% \mathrm{w} / \mathrm{v}$ aqueous solution of PVA under constant stirring

Table 1

Materials used in dispensing system.

\begin{tabular}{lcccc}
\hline Polymer type & $\begin{array}{c}2.23 \% \\
\text { Glutaraldehyde } \\
\text { solution in water } \\
\text { (\% crosslinked) }\end{array}$ & $\begin{array}{c}6.25 \% \\
\text { Glutaraldehyde } \\
\text { solution in water } \\
(\% \text { crosslinked })\end{array}$ & $\begin{array}{c}\text { PVA 10\% w/v } \\
\text { aqueous solution } \\
(\mathrm{ml})\end{array}$ & $\begin{array}{c}\text { Chitosan } 1.3 \% \mathrm{w} / \mathrm{v} \\
\text { dissolved in } 2 \% \mathrm{v} / \mathrm{v} \\
\text { acetic acid }(\mathrm{ml})\end{array}$ \\
\hline PVA gel & 5 & $-\overline{7}$ & 10 & - \\
PVA/chitosan & - & 10 & 10 \\
\hline
\end{tabular}


for $30 \mathrm{~min}$ at room temperature. The biopolymer solution is made from $1.3 \% \mathrm{w} / \mathrm{v}$ chitosan dissolved in $2 \% \mathrm{v} / \mathrm{v}$ acetic acid and $10 \% \mathrm{w} / \mathrm{v}$ aqueous solution of PVA. The weight ratio of the PVA/chitosan mixture was adjusted to $1: 1$, then $7 \%$ of the crosslinking solution $(6.25 \%$ glutaraldehyde in water) was added and mixed under 30 min constant stirring.

\section{Experimental Setup}

Figure 1(a) shows a photograph taken from the experimental setup. In Fig. 1(b), the diagram presents the experimental setup, which is the combination of a fluid dispensing system, a system for measuring syringe pressure, a compressed air pressure regulator system, and a vision measurement system. These systems are controlled by a single program in which it functions as an intelligent measurement system and a compressed air pressure control system. A fluid dispensing system comprises a syringe with a needle attached and a compressed air supply line. An Iwashita AD2000 dispenser was modified to support a computer programing command via an in-house fabricated control board. Command signals to the dispenser were synchronized among all the system components. As soon as the fluid begins to be dispensed, the pressure measurement system and the vision measurement system collect data instantaneously.

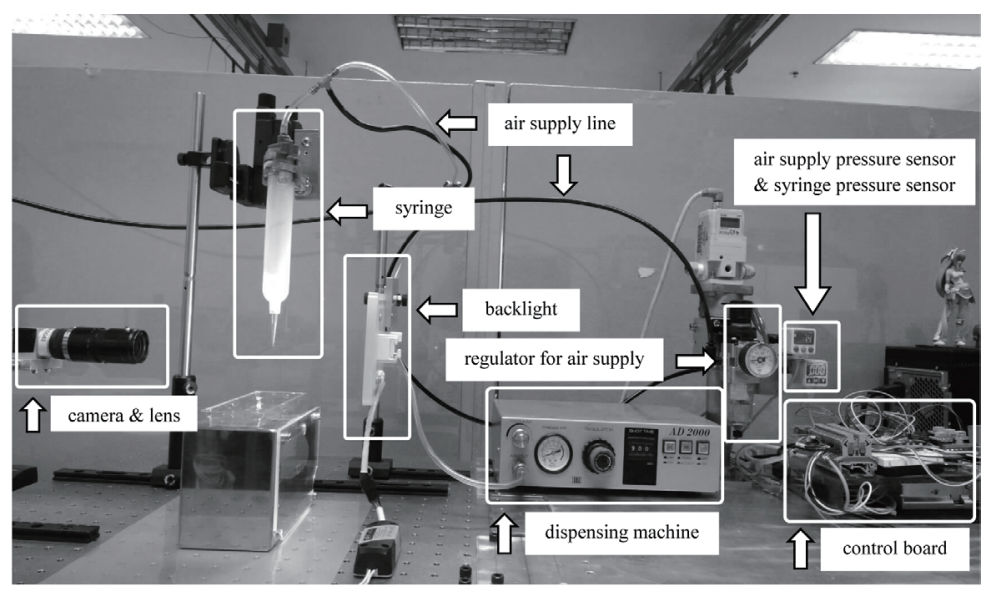

(a)

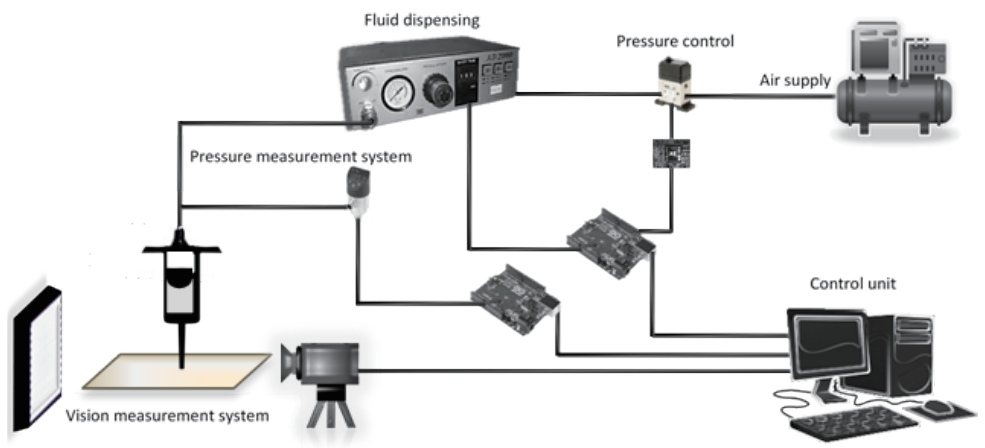

(b)

Fig. 1. (Color online) (a) Real experimental setup and (b) experimental schematic. 
A pressure sensor (brand SMC) was installed in the compressed air transmission line between the syringe and the dispenser to measure the pressure inside the syringe. The recorded pressure signals are also used to determine the actual pressure supply that is controlled by a pressure control system. The compressed air pressure control system includes a voltage control regulator, a compressed air transmission line, and a control board that receives commands from the control unit. The vision measurement system consists of a charge-couple device (CCD) camera and a backlight installed at the opposite side of the camera. The camera was used to record an image of the fluid being dispensed from a needle attached to the syringe. The photographs of the dispensed fluid are then used in a method for determining the volume as described in the following section.

\subsection{Experimental procedure}

Figure 2 shows the experimental procedure. The setting of initial conditions is initially performed in the control unit. Subsequently, a user command is used to activate the program to perform the dispensing process with the pressure measurement system and vision measurement system simultaneously. The compressed air pressure in the syringe is measured and recorded; meanwhile, the vision measurement system records the dispensed fluid images and sends them to the buffer for further analysis and volume calculation. Subsequently, in the data analysis procedure, the fluid flow rates of the measured volumes were used to construct a pressuredependent fluid flow rate curve as a function of time to identify the rheological properties of the fluids.

\subsection{Dispensed droplet image visualization and volume estimation from captured images}

For droplet image visualization, a region of interest (ROI), pixel-to-length ratio and process runtime were configured for the program initial setup, shown in Figs. 3(a) and 3(b). The photograph of the graphic user interface (GUI) for measurement and control program is shown

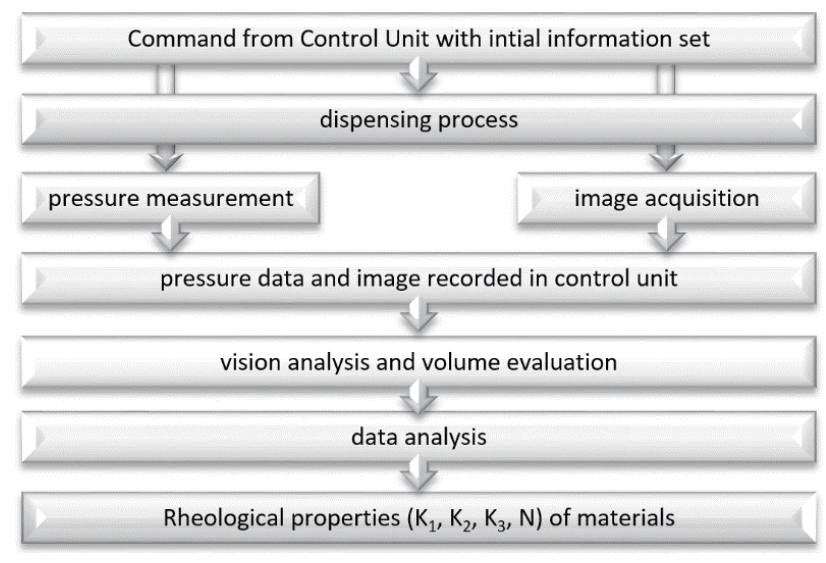

Fig. 2. Experimental procedure. 


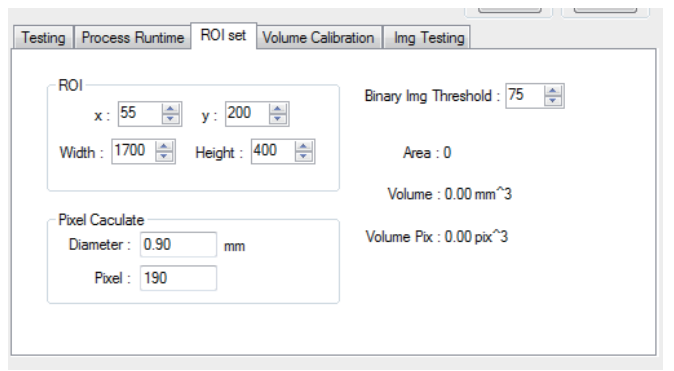

(a)

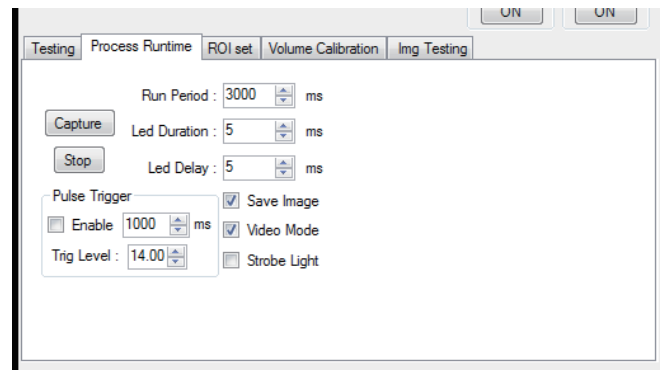

(b)

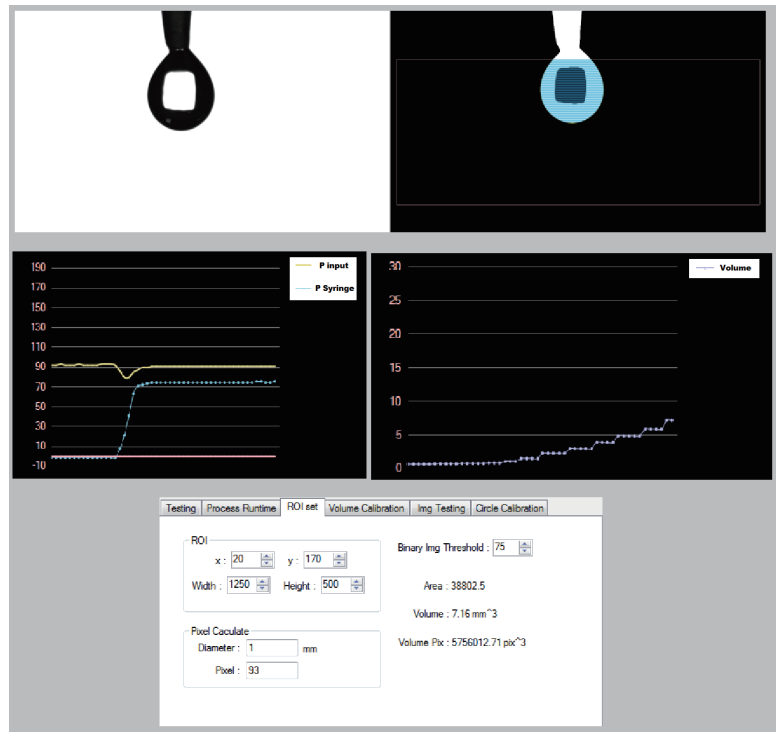

(c)

Fig. 3. (Color online) (a) ROI identification, (b) setup of the dispensing time duration, and (c) multiple views of the program.

in Fig. 3(c). To acquire an image, a CCD camera is always operated in video mode with a backlight turned on. A command signal from the program activates the dispensing machine and synchronously takes a photograph of the dispensed fluid for the extent of the dispensing process. The typical captured image of a fluid droplet is shown in Fig. 4(a). Then a binary image analysis algorithm is used to detect the edge of the dispensed fluid and record the detected edge as a contour as shown in Fig. 4(b). The image contour is used for the volume evaluation described below.

The dispensed fluid through the needle is assumed to be symmetrical; thus, the volume of the three-dimensional dispensed fluid can be evaluated using the contour of the two-dimensional image obtained from a binary image analysis. An image contour is sliced horizontally into a cylindrical shape similar to a rotational body. ${ }^{(2)}$ The height of the cylindrical slice is set equal to 1 pixel and the length is determined by defining the edge of an image contour. The unit of pixel is converted into scale described in mm using the pixel-to-length ratio set in the program as an initial condition. The volume of each cylindrical slice can be calculated using Eq. (1), 


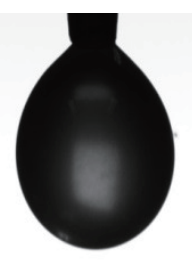

(a)

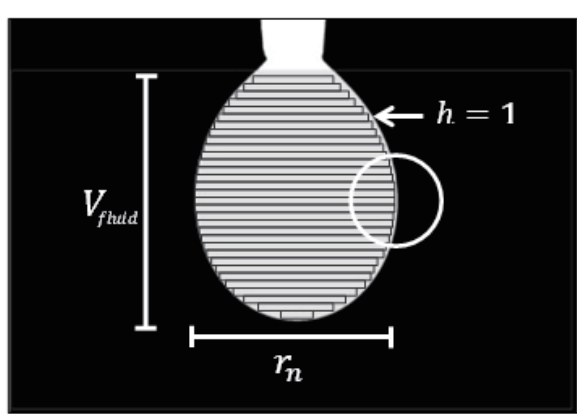

(c)

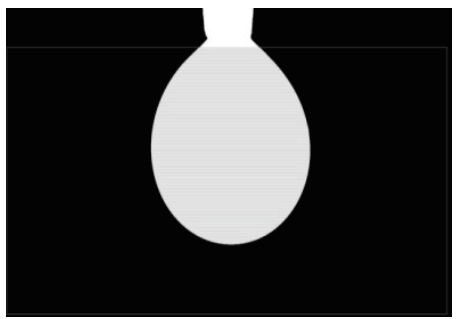

(b)

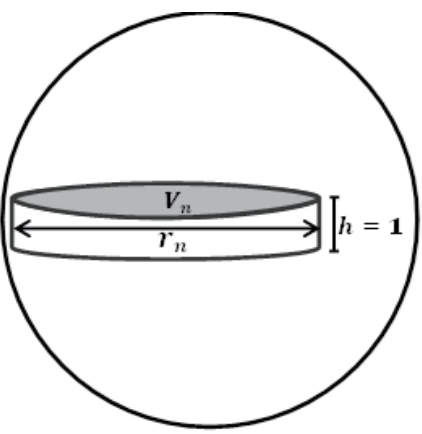

Fig. 4. (a) Picture of the droplet, (b) image contour after performing the binary image analysis algorithm, and (c) a slice of the image contour.

$$
V_{n}=\pi r_{n}^{2} h
$$

where $V_{n}$ is the volume of each slice at number $n, n$ is the number of slices that equal the height of the rotational body divided by $h, h$ is the height of one cylindrical slice, the unit of $h$ is pixels, $H$ is the height of the rotational body, and $r$ is the radius of the sliced rotational body, which is equal to half of the cylindrical length. Therefore, the volume of a three-dimensional dispensed fluid can be evaluated using Eq. (2).

$$
V_{\text {fluid }}=\sum_{n=1}^{H} V_{\text {fluid }}=\sum_{n=1}^{H} \pi r_{n}^{2} h
$$

Equation (2) is applied to the image contour in Fig. 4(b), then the droplet volume can be evaluated.

\subsection{Droplet volume calibration}

In order to evaluate the accuracy of the proposed measurement method, the standard bearing balls with diameters of 1.0 and $2.0 \mathrm{~mm}$ are adopted. The ball simulates the droplet being dispensed with calculable volume with high accuracy. The syringe with needle, camera, and backlight are positioned comparable to the actual dispensing experimental setup. The ball is carefully attached to a tip of the needle by a clear adhesive. The program activates the 
camera to capture the ball image as shown in Fig. 5(a). Then a binary image analysis algorithm described previously was performed to detect the edge of the bearing ball and record it as a contour image shown in Fig. 5(b). The volume of the bearing ball can be evaluated using Eq. (2) and shown in Fig. 5(b). To calibrate a measured volume, this procedure is repeated several times for each diameter of the bearing balls. The comparison between the calculated volume and the measured volume is shown in Table 2. The measured volume of the ball of $1.0 \mathrm{~mm}$ diameter shows a standard deviation of $0.05 \mathrm{~mm}^{3}$ and that of the ball of $2.0 \mathrm{~mm}$ diameter shows a standard deviation of $0.45 \mathrm{~mm}^{3}$. It is clear that our method shows a good relationship between the standard volume and the measured volume.

\subsection{Determination of rheological properties of dispensed fluid}

To determine rheological properties, the modeling of a fluid dispensing process is required. Many researchers ${ }^{(2-7)}$ have studied fluid dispensing models to characterize the flow behavior of a process. Because a biopolymer is a non-Newtonian fluid, the relationship between the shear stress and the shear rate is nonlinear. For a non-Newtonian fluid with a zero yield stress, the power law in Eq. (3) is applied:

$$
Q=K_{0} \Delta p^{N}
$$

However, the yield stress of a non-Newtonian fluid sometimes does not equal zero; therefore, the generalized power law is the more acceptable method. Hence, Eq. (4) presents

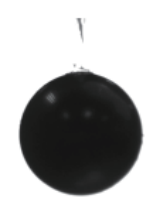

(a)

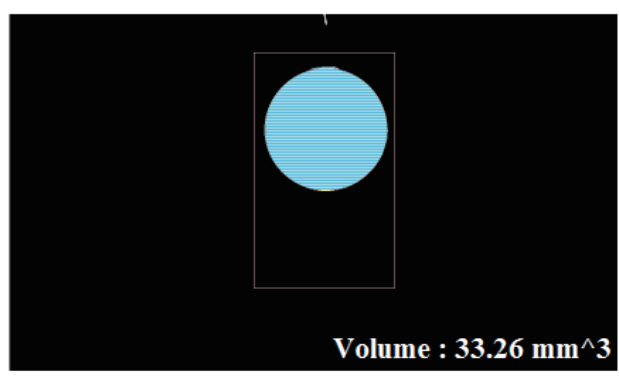

(b)

Fig. 5. (Color online) Volume calibration of the bearing ball of $2 \mathrm{~mm}$ diameter: (a) a picture of the bearing ball and (b) an image contour of the bearing ball picture with the volume presented.

Table 2

A comparison between calculated volume and measured volume of the bearing ball.

\begin{tabular}{lcc}
\hline $\begin{array}{l}\text { Standard bearing ball size } \\
\text { (radius) }\end{array}$ & $\begin{array}{c}\text { Calculated volume }\left(\mathrm{mm}^{3}\right) \\
\text { from ball sizes }\end{array}$ & $\begin{array}{c}\text { Measured volume }\left(\mathrm{mm}^{3}\right) \\
\text { using our method }\end{array}$ \\
\hline $1 \mathrm{~mm}$ & 4.18 & $4.11 \pm 0.05$ \\
$2 \mathrm{~mm}$ & 33.51 & $33.11 \pm 0.45$ \\
\hline
\end{tabular}


the relationship between the fluid flow rate and the syringe pressure in one dispensing cycle in terms of $\Delta p$ :

$$
Q=K_{1} \Delta p^{N}+K_{2} \Delta p^{N-1}+K_{3} \Delta p^{N-2},
$$

where $N=1 / n$ and the coefficients $K_{1}, K_{2}$, and $K_{3}$ are ${ }^{(2)}$

$$
\begin{gathered}
K_{1}=\frac{2 L \pi n R^{2} C^{1+(1 / n)}}{k^{1 / n}(3 n+1)}, \\
K_{2}=\frac{8 L \pi n^{2} R \tau_{0} C^{1+(1 / n)}}{k^{1 / n}(2 n+1)(3 n+1)}, \\
K_{3}=\frac{16 L \pi n^{3} R \tau_{0}^{2} C^{1+(1 / n)}}{k^{1 / n}(n+1)(2 n+1)(3 n+1)},
\end{gathered}
$$

where $k$ is the fluid viscosity, $n$ is the power law or flow behavior index, $C=\left(R-r_{0}\right) / 2 L$, and $R$ is the radius of the syringe. The term $r_{0}$ is the critical diameter for which $r_{0}=2 \tau_{0} L / \Delta p$ and $\tau_{0}$ is the yield stress of liquid. Therefore, the rheological properties of the material were determined by numerically fitting the generalized power law [Eq. (4)] by a similar procedure. ${ }^{(2)}$

\section{Results and Discussion}

The experiments were carried out using the aqueous solutions of PVA and PVA/CS. They are widely known as non-Newtonian materials. Their rheological properties gradually evolved after being mixed and put in the syringe during the dispensing process within 50 min. Figure 6(a) shows a sequence of dispensed fluid volume profiles at increments of $100 \mathrm{~ms}$. Each image in Fig. 6(a) is processed with the procedure described above to evaluate each droplet volume. Figure 6(b) shows the plot of a correspondeing measured volume of the fluid versus time at increments of $100 \mathrm{~ms}$. Figure 6(c) shows a recorded dispensing pressure of the fluid plotted versus the same time scale. Evidently, the increase in syringe pressure increases the fluid droplet volume. For different time stamps and recorded applied pressures, the measured flow rates were used to construct pressure-dependent fluid flow rate curves every 10 min for 6 experiments in the case of PVA (within $60 \mathrm{~min}$ ). In the case of PVA/CS, the curves were constructed at different sampling times for only 4 experiments (within $10 \mathrm{~min}$ ). This is because the viscosity of PVA/CS promptly changed owing to the high-level gelation phenomena. The resulting curves are shown in Fig. 7 for PVA and Fig. 8 for PVA/CS. The evolution of viscoelasticity during the gelation reactions of PVA in the presence of a cross-linker (glutaraldehyde) is the time-dependent growth of viscoelasticity similar to the results reported 


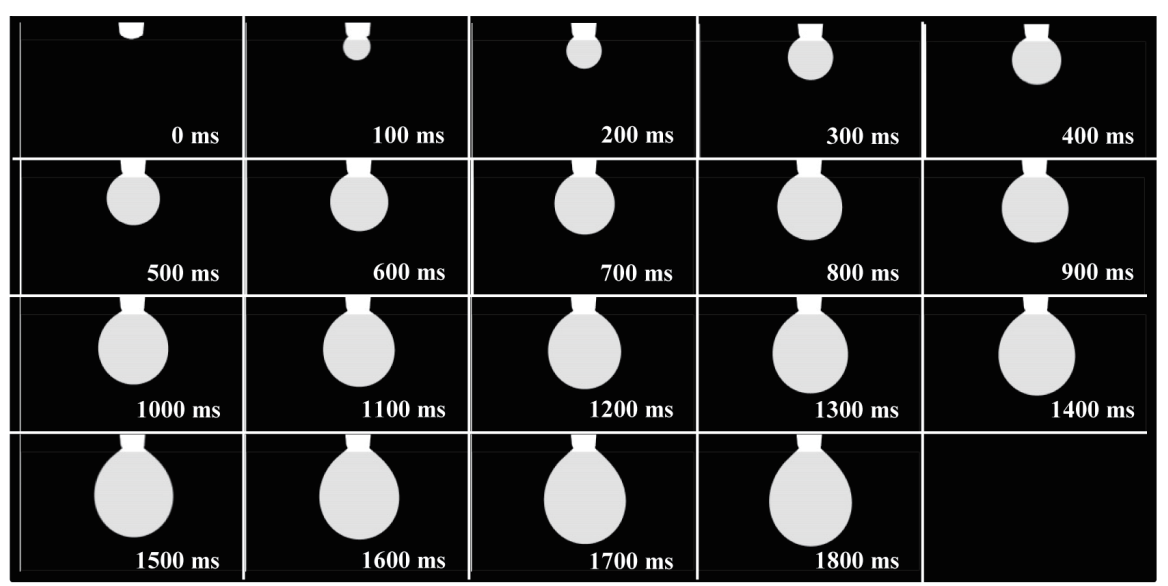

(a)

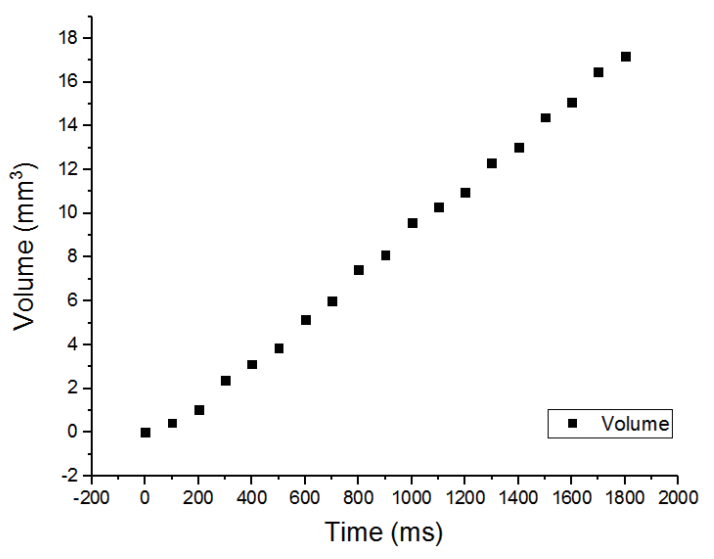

(b)

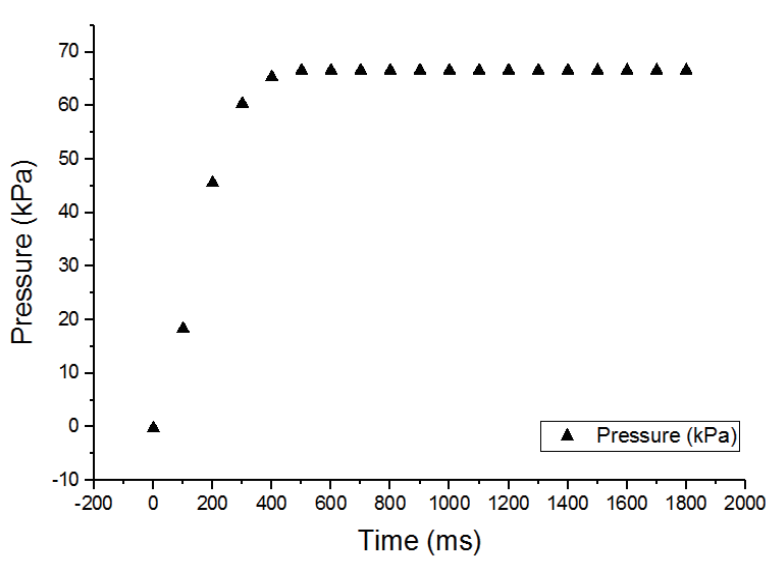

(c)

Fig. 6. (a) A sequence of dispensed fluid volume profiles at increments of $100 \mathrm{~ms}$, (b) a volume of the fluid at increments of $100 \mathrm{~ms}$, and (c) a dispensing pressure of the fluid at increments of $100 \mathrm{~ms}$.

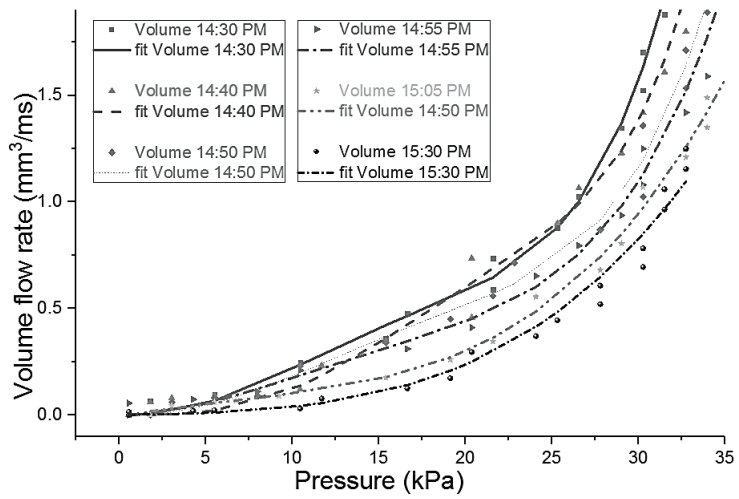

Fig. 7. Pressure-dependent fluid flow rate curve of PVA and the numerical fitting curve (solid line) determined using generalized power law for modeling a fluid dispensing process [Eq. (4)].

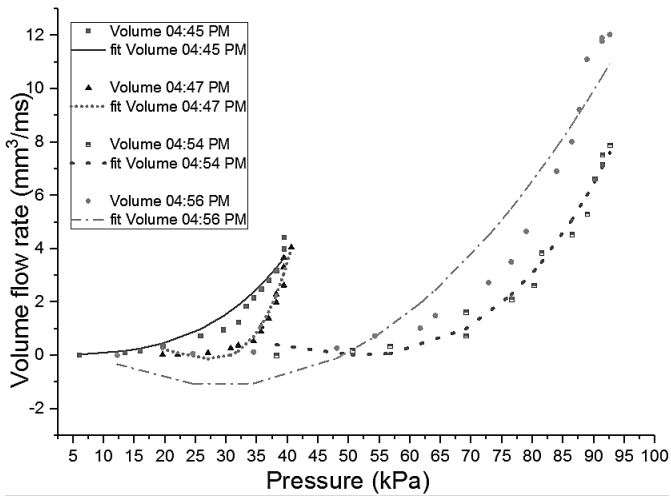

Fig. 8. Pressure-dependent fluid flow rate curve of $\mathrm{PVA} / \mathrm{CS}$ and the numerical fitting curve (solid line) determined using generalized power law for modeling a fluid dispensing process [Eq. (4)]. 
in the literature. ${ }^{(12)}$ According to the pressure-dependent fluid flow rate curve, the fluid flows of both PVA and PVA/CS are significantly sensitive to time changes. With these results, it is clear that the real-time rheological properties measurement system is critically needed to identify fluid flow behavior at a specific time during the fluid dispensing process.

To quantify the experimental results, the rheological properties of the material were determined by numerically fitting the generalized power law in Eq. (4). The resulting values of $K_{1}, K_{2}, K_{3}$, and $N$ are tabulated in Table 3 for PVA and Table 4 for PVA/CS. Figure 9 illustrates the measured $N$ values of both materials and the curve fitting results. The $N$ value is the inverse function of the power law index $n$. The value of $n$ is primarily influenced by the rheological properties of the fluid instead of the process parameters. The higher the power law index $n$, the lower the calculated $N$. Therefore, the resulting $N$ values gradually decrease with increasing dispensing time. These results are comparable to the actual behavior of dispensed fluids in which they become more viscous (increasing $n$ ) after they are mixed and injected from the syringe. However, for $K_{1}, K_{2}$, and $K_{3}$, no significant change is observed in this study.

Table 3

Rheological properties of PVA.

\begin{tabular}{lccll}
\hline Time examined & $K_{1}$ & \multicolumn{1}{c}{$K_{2}$} & \multicolumn{1}{c}{$K_{3}$} & \multicolumn{1}{c}{$N$} \\
\hline 14:30 PM & $1.54 \mathrm{E}-06$ & $-7.82 \mathrm{E}-05$ & 0.001204 & 5.46 \\
14:40 PM & $6.94 \mathrm{E}-08$ & $-4.18 \mathrm{E}-06$ & $7.18 \mathrm{E}-05$ & 5.18 \\
14:50 PM & $9.97 \mathrm{E}-07$ & $-5.46 \mathrm{E}-05$ & 0.0009112 & 4.593 \\
$14: 55$ PM & $2.67 \mathrm{E}-06$ & -0.0001313 & 0.002206 & 4.177 \\
$15: 05$ PM & $2.51 \mathrm{E}-05$ & -0.0006048 & 0.009258 & 3.244 \\
15:30 PM & $2.00 \mathrm{E}-05$ & $4.36 \mathrm{E}-06$ & $7.47 \mathrm{E}-07$ & 2.824 \\
\hline
\end{tabular}

Table 4

Rheological properties of PVA/CS.

\begin{tabular}{lcrll}
\hline Time examined & \multicolumn{1}{c}{$K_{1}$} & \multicolumn{1}{c}{$K_{2}$} & \multicolumn{1}{c}{$K_{3}$} & \multicolumn{1}{c}{$N$} \\
\hline $04: 45$ PM & $1.10 \mathrm{E}-05$ & $2.32 \mathrm{E}-10$ & 0.002236 & 5.426 \\
04:47 PM & $1.43 \mathrm{E}-05$ & $-7.77 \mathrm{E}-04$ & 0.01038 & 4 \\
04:54 PM & $5.06 \mathrm{E}-05$ & -0.005304 & 0.1392 & 3 \\
04:56 PM & 0.002307 & $-1.33 \mathrm{E}-01$ & 1 & 2.057 \\
\hline
\end{tabular}

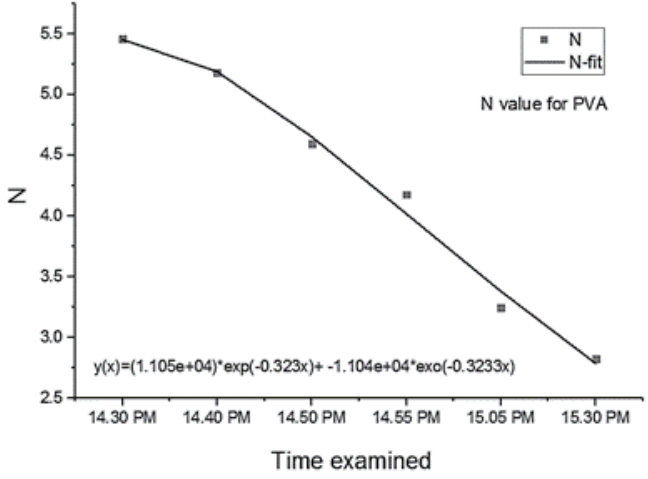

(a)

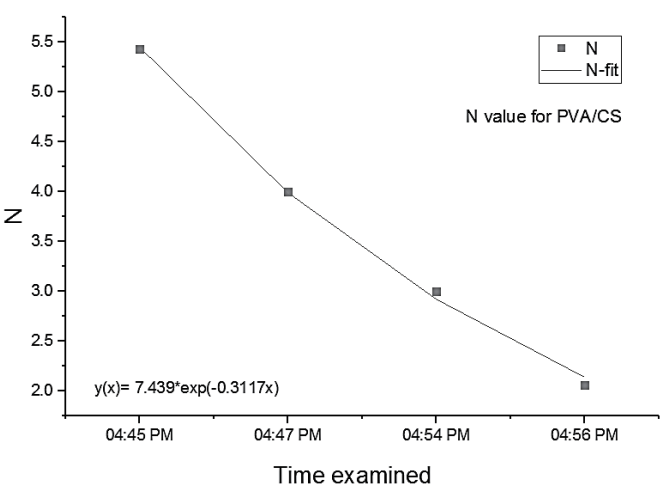

(b)

Fig. 9. Graph of $N$ for (a) PVA and (b) PVA/CS. 


\section{Conclusions}

The innovative rheological properties measurement system presented herein was developed to provide satisfactory rheological parameters of materials under certain processing conditions. Because the biopolymers are highly time-dependent, a traditional rheological properties measurement system may yield inaccurate information. The proposed measurement system is a combination of computational programming with a physical sensor measurement system. The system aims to provide a simple user interface to control the dispensing process and perform data analysis to determine the rheological properties of materials simultaneously. Therefore, this system is better than the conventional method in which the rheological properties of materials should be measured by a separate and expensive rheometer. The system utilizes an image processing procedure, a physical sensor system, and numerical flow modeling to characterize the flow behavior of a dispensed fluid. The results reveal that the value of $N$ changes significantly according to the rheological properties of the fluids. They are comparable to the actual behavior of dispensed fluids in which they become more viscous (increasing $n$ ) after they are mixed and injected from the syringe. The resulting $N$ can be used to perform automatic fluid flow control in future research.

\section{References}

1 X. Li, R. Cui, L. Sun, K. Aifantis, Y. Fan, Q. Feng, F. Cui, and F. Watari: Int. J. Polym. Sci. 2014 (2014) 1. https://doi.org/10.1155/2014/829145

2 S. Boonsang and W. Lertkittiwattanakul: Measurement 102 (2017) 57. https://doi.org/10.1016/ j.measurement.2017.01.050

3 H. X. Li, J. Liu, C. P. Chen, and H. Deng: IEEE/ASME Trans. Mechatron. 12 (2007) 491. https://doi. org/10.1109/TMECH.2007.901946

4 X. B. Chen, W. J. Zhang, G. Schoenau, and B. Surgenor: IEEE Trans Electron. Packag. Manuf. 26 (2003) 286. https://doi.org/10.1109/TEPM.2003.820824

5 Y.-X. Zhao and X.-D. Chen: Int. J. Adv. Manuf. Technol. 55 (2011) 433. https://doi.org/10.1007/s00170-010$3108-2$

6 K. Y. Chan and C. K. Kwong: Int. J. Adv. Manuf. Technol. 65 (2013) 589. https://doi.org/10.1007/s00170-0124202-4

7 X. B. Chen: Int. J. Adv. Manuf. Technol. 43 (2009) 276. https://doi.org/10.1007/s00170-008-1700-5

8 K. Thurow, T. Krüger, and N. Stoll: J. Autom. Methods Manage. Chem. 2009 (2009) 198732. https://doi. org/10.1155/2009/198732

9 X. Lu and M. Huang: Int. J. Adv. Manuf. Technol. 75 (2014) 1197. https://doi.org/10.1007/s00170-014-6188-6

10 N. He, T. Liu, and B. Liu: J. Nanosci. Nanotechnol. 16 (2016) 58. https://doi.org/10.1166/jnn.2016.11681

11 Q. H. Nguyen and S. B. Choi: IEEE Trans. Electron. Packag. Manuf. 31 (2008) 134. https://doi.org/10.1109/ TEPM.2008.919348

12 A. Izuka, H. H. Winter, and T. Hashimoto: Macromolecules 25 (1992) 2422. https://doi.org/10.1021/ ma00035a020 